\title{
Radiometric monitoring of atmospheric water vapor as it pertains to phase correction in millimeter interferometry
}

\author{
E.C. Sutton and R.M. Hueckstaedt ${ }^{\star}$ \\ Department of Astronomy, University of Illinois, 1002 W. Green St., Urbana, IL 61801, U.S.A.
}

Received November 21, 1995; accepted March 26, 1996

\begin{abstract}
Water vapor in the Earth's troposphere produces fluctuations in the phase of millimeter-wavelength radiation from astronomical sources. Such fluctuations seriously limit the spatial resolution achievable with current millimeter interferometers. Since water vapor is also a source of atmospheric opacity at these wavelengths, radiometric measurements of sky brightness may be used to monitor the fluctuating water vapor content of the atmosphere and thereby the fluctuations in the interferometric phase. The atmospheric opacity depends on the frequency and on the physical conditions of those atmospheric regions in which the water vapor is located. Atmospheric temperature influences the strengths of the various absorption lines, and pressure influences the degree of line broadening. The magnitude of the phase fluctuations relative to the brightness fluctuations is therefore also dependent on frequency, temperature, and pressure. The frequency of a radiometric monitoring system may be chosen to minimize the dependence of this ratio on the atmospheric parameters.
\end{abstract}

Key words: atmospheric effects — instrumentation: interferometers — methods: observational — techniques: interferometric

\section{Introduction}

The spatial resolution achievable with millimeterwavelength interferometry is often limited by the Earth's atmosphere. Inhomogeneities in the atmosphere introduce random fluctuations in the phases of signals from astronomical sources. These fluctuations can be rather large at millimeter wavelengths and often correspond to phase differences exceeding 1 radian for baselines longer than about 100 meters. Such fluctuations seriously degrade the spatial resolution and the positional accuracy of interferometric measurements and also limit their sensitivity.

A variety of schemes can be employed to measure and thereby compensate for such phase fluctuations. With most interferometers the phase is calibrated using signals from nearby point sources. This procedure is effective at correcting for baseline errors and other instrumental effects, but is generally ineffective at removing atmospheric fluctuations (except those with very long time scales). For bright sources self-calibration techniques may be very effective at removing atmospheric phase errors. But for fainter sources such errors remain a problem. The phase fluctuations at millimeter wavelengths are largely due to

Send offprint requests to: E.C. Sutton (sutton@astro.uiuc.edu) ${ }^{\star}$ Currently with Astronomy Department, University of Florida, P.O. Box 112055, 211 SSRB, Gainesville, FL 32611-2055, U.S.A. tropospheric water vapor. Since water vapor is also a significant source of opacity at these wavelengths, fluctuations in the sky brightness may be used to infer the corresponding phase fluctuations. In this paper we discuss some of the issues relating to radiometric monitoring of atmospheric water vapor and the possibility of using radiometric measurements to correct for atmospheric phase fluctuations.

\section{Physics of atmospheric absorption and phase delay}

\subsection{Line absorption by water vapor}

This work is concerned with the absorption and phase delay produced by atmospheric water vapor at frequencies up to $1000 \mathrm{GHz}$. A good general introduction to this topic has been given by Waters (1976). In these calculations we neglect absorption produced directly by molecular oxygen and other atmospheric gases. However, oxygen and nitrogen contribute to the pressure broadening of the water vapor lines since they are the primary collisional partners of the water molecules. We also neglect absorption by droplets of liquid water.

The spectrum of water vapor contains hundreds of strong lines throughout the submillimeter and far-infrared spectral regions. The structure of the water molecule is 
well known and its line frequencies, strengths, and excitation energies are well determined. In this work we use a data base of 424 lines compiled by Poynter \& Pickett (1984). Each water line is characterized by a resonant frequency $\nu_{0}$, a line strength $S$, a spin degeneracy $g$, and upper and lower state energies $E_{\mathrm{u}}$ and $E_{\mathrm{l}}$. Roughly 100 of the lines in this compilation make negligible contributions to the atmospheric absorption due to either their low line strengths or their high excitation energies.

In terms of these molecular parameters, the absorption coefficient at a frequency $\nu$ is given by

$$
\begin{aligned}
\alpha= & \frac{8 \pi^{3}}{3 h c} \nu \sum_{i} \frac{\mu^{2} g_{i} S_{i}}{Q} n_{\mathrm{H}_{2} \mathrm{O}}\left(\mathrm{e}^{-E_{l i} / k T}-\mathrm{e}^{-E_{u i} / k T}\right) \\
& \phi\left(\nu, \nu_{0 i}, \gamma_{i}\right) .
\end{aligned}
$$

In this expression $\mu$ is the permanent dipole moment of water $(1.8546 \mathrm{D}), n_{\mathrm{H}_{2} \mathrm{O}}$ is the number density of water molecules, and the sum is over the set of absorption lines. It is assumed that the molecular levels are populated in accordance with a Boltzmann distribution at a temperature $T$. The line shape $\phi\left(\nu, \nu_{0 i}, \gamma_{i}\right)$ will be discussed below. The rotational partition function $Q$ is adequately approximated near room temperature by the expression $172.2 \times(T / 293)^{1.5}$, based on the values tabulated by Poynter \& Pickett (1984). This is in good agreement with an earlier calculation of $Q$ by Gaut (1968) and with the analytical form for the high temperature limit (Herzberg 1945).

When integrated over a short atmospheric path of length $l$ with constant physical conditions this leads to an opacity

$$
\begin{aligned}
\tau & =\frac{8 \pi^{3}}{3 h c} \nu \sum_{i} \frac{\mu^{2} g_{i} S_{i}}{Q} \rho_{w} w \frac{N_{\mathrm{A}}}{m_{\mathrm{H}_{2} \mathrm{O}}}\left(\mathrm{e}^{-E_{l i} / k T}-\mathrm{e}^{-E_{u i} / k T}\right) \\
& \phi\left(\nu, \nu_{0 i}, \gamma_{i}\right)
\end{aligned}
$$

where $w$ is the precipitable water vapor content of that path, defined by

$$
w=\frac{1}{\rho_{w}} \rho_{v} l .
$$

Here $\rho_{w}$ is the mass density of liquid water $\left(1 \mathrm{~g} \mathrm{~cm}^{-3}\right)$, $N_{\mathrm{A}}$ is Avogadro's number, and $m_{\mathrm{H}_{2} \mathrm{O}}$ is the atomic mass of water.

The absorption spectrum and the phase delay introduced by the water vapor are related via the KramersKronig transform. Often this is expressed in terms of a relationship between the real and imaginary parts of the index of refraction,

$$
n_{\mathrm{r}}(\omega)-1=\frac{2}{\pi} P \int_{0}^{\infty} \frac{\Omega n_{i}(\Omega)}{\Omega^{2}-\omega^{2}} \mathrm{~d} \Omega
$$

where $P$ represents the Cauchy principal value. Applying the Kramers-Kronig transform to the above absorption spectrum leads to a phase delay spectrum (expressed as an equivalent delay length $L$ ),

$$
\begin{aligned}
L= & \frac{1}{2 \pi} \frac{8 \pi^{3}}{3 h c} \sum_{i} \frac{\mu^{2} g_{i} S_{i}}{Q} \rho_{w} w \frac{N_{\mathrm{A}}}{m_{\mathrm{H}_{2} \mathrm{O}}}\left(\mathrm{e}^{-E_{l i} / k T}-\mathrm{e}^{-E_{u i} / k T}\right) \frac{c}{2} \\
& \phi_{\mathrm{KK}}\left(\nu, \nu_{0 i}, \gamma_{i}\right) .
\end{aligned}
$$

The quantity $\phi_{\mathrm{KK}}$ is the appropriate Kramers-Kronig transform of the line shape $\phi$.

\subsection{Line shape theory}

In this work we adopt the kinetic or "Gross" collisional line shape (Gross 1955). The earlier collisional line shape of van Vleck \& Weisskopf (1945) is known to underestimate absorption strengths in the line wings by factors of order 4 (van Vleck 1947). In contrast, the kinetic line shape gives significantly better agreement with experimental data and is based on a more reasonable physical picture of the collisional interactions. The van Vleck - Weisskopf model required rapid rearrangements of molecular orientations which, for instantaneous collisions, needed to occur at infinite velocity.

The kinetic line shape is specified by

$$
\phi(\nu)=\frac{1}{\pi} \frac{4 \nu \nu_{0} \gamma}{\left(\nu_{0}^{2}-\nu^{2}\right)^{2}+4 \nu^{2} \gamma^{2}},
$$

where $\nu_{0}$ is the resonance frequency and $\gamma$ is the frequency half width at half maximum (HWHM). The corresponding Kramers-Kronig shape factor, which enters into the phase delay, is given by

$$
\phi_{\mathrm{KK}}(\nu)=\frac{2}{\pi} \nu_{0} \frac{\nu_{0}^{2}-\nu^{2}}{\left(\nu_{0}^{2}-\nu^{2}\right)^{2}+4 \nu^{2} \gamma^{2}} .
$$

The line shape formulae in Eqs. (6) and (7) are specifically written to be compatible with Eqs. (1), (2), and (5). The literature on line shape theory contains an unfortunate number of errors and misapplications of many of the basic line shape formulae. Falcone (1969) has helped clarify the situation.

The kinetic line shape formula is likely to break down at sufficiently large frequency shifts away from line center. The Gross theory is based on instantaneous interactions. The effect of a finite collisional time will be to modify the line shape at shifts from line center of order the reciprocal of the collisional time. Ben-Reuven (1969) has derived a more general theory, which yields both the van Vleck - Weisskopf and the Gross results as limiting cases. The Ben-Reuven theory contains free parameters which are not, in general, known. However, it appears that for collisional broadening in the Earth's atmosphere, a result somewhat near the kinetic line shape should be obtained.

The line width parameter $\gamma$ is an important aspect of the line shape theory. For foreign gas broadening (collisions with air molecules but not other water molecules) 
the line width varies with pressure and temperature as

$$
\gamma=\gamma_{0}\left(\frac{P}{1013.25}\right)\left(\frac{300}{T}\right)^{x}
$$

where $P$ is the pressure in $\mathrm{mb}$ and $T$ is the temperature in Kelvin. For the ten lowest frequency water lines in our list, the widths $\gamma_{0}$ and the temperature coefficients $x$ are taken from Waters (1976). For 19 additional lines the widths are taken from Liebe (1981) and Burch (1968), with the temperature coefficient set at $x=0.62$. All remaining lines are assumed to have $\gamma_{0}=2.63 \mathrm{GHz}$ and $x=0.62$, the average values for those quantities in the calculations of Benedict \& Kaplan (1959).

Water vapor molecules also undergo collisions with other water molecules, which are roughly five times as effective at collisional broadening than either molecular nitrogen or oxygen. For simplicity we have increased the widths determined above by a factor of 1.025 to account for self broadening. This factor is appropriate for typical conditions at an altitude of a few $\mathrm{km}$ above sea level. The error introduced by this approximation will be negligible except when one needs to describe humid conditions at low altitude.

\subsection{Excess absorption}

Calculations of the sort indicated above lead to synthetic atmospheric absorption spectra which tend to underestimate the observed absorption in regions of good atmospheric transparency (Waters 1976). This has suggested the possible existence of an "excess absorption" mechanism, variously attributed to the presence of water vapor dimers or to possible inadequacies in the spectroscopic theory. Zammit \& Ade (1981) report that this excess depends linearly on water vapor content, suggestive of the latter possibility. Calculations indicate that minor atmospheric constituents, such as ozone, provide only small amounts of absorption except at certain specific frequencies (Waters 1976). As discussed above, use of the kinetic (Gross) line shape is an important factor in minimizing the discrepancy between observation and theory. Nevertheless, any remaining "excess absorption" must be fully understood, as it affects both the absorption and the phase delay.

One frequently used empirical formula for the excess absorption was introduced by Gaut \& Reifenstein (1971). Expressed in terms of an excess opacity their formula is

$$
\Delta \tau(\nu)=1.0810^{-5} w\left(\frac{300}{T}\right)^{2.1}\left(\frac{P}{1000}\right)\left(\frac{\nu}{10^{9}}\right)^{2}
$$

where $P$ is the pressure in $\mathrm{mb}, T$ is the temperature in Kelvin, $\nu$ is the frequency in $\mathrm{Hz}$, and $w$ is the precipitable water vapor content in $\mathrm{cm}$. Their excess absorption coefficient is proportional to the square of the frequency of observation. Such a term, however, is unphysical and in this context is particularly troublesome since it introduces a divergence in the calculation of the phase delay. An arbitrary upper frequency cutoff may be provided to prevent this divergence, but the absorption spectrum remains physically unappealing and the low frequency phase delay is sensitive to the choice of cutoff frequency.

A different formula for the supposed excess was given by Zammit \& Ade (1981),

$$
\Delta \tau(\nu)=5.2710^{-4} w\left(\frac{280}{T}\right)^{2.1}\left(\frac{P}{850}\right)\left(\frac{\nu}{10^{9}}\right)^{1.22} .
$$

With a frequency dependence of $\nu^{1.22}$, the divergence in the theory remains. But here it is possible to introduce an upper frequency cutoff of order $1000 \mathrm{GHz}$, in which case most observable parameters will be rather insensitive to the exact cutoff frequency. It is interesting to note that Zammit and Ade suggest that inadequacies in the line shape theory are the cause of this excess, in which case the excess should be terminated near the resonance frequencies of the strong sub-millimeter water lines. The magnitude of the Zammit and Ade excess exceeds that of Gaut and Reifenstein at centimeter and longer wavelengths but falls below that of Gaut and Reifenstein at submillimeter wavelengths. Our calculations have been done without any excess and with both the Gaut and Reifenstein and the Zammit and Ade forms of the excess. In each case in which excess absorption has been considered, we limit the excess to frequencies below $1000 \mathrm{GHz}$.

\subsection{Comparison with measured absorption coefficients}

We have compared our theoretical absorption coefficients with various measured values discussed by Burch (1968), Waters (1976), Liebe (1984), Liebe (1985), and Danese \& Partridge (1989). It appears as if some form of "excess absorption" is needed to adequately fit the experimental data, particularly in the lower frequency atmospheric windows. However the Zammit and Ade formula clearly overstates the absorption coefficient at frequencies below $100 \mathrm{GHz}$ and the Gaut and Reifenstein formula similarly overstates the absorption coefficient at frequencies near $1000 \mathrm{GHz}$. It appears as if no simple power law excess is consistent with the entire set of experimental data. We regard absorption coefficients and opacities derived using these two forms of the excess absorption as upper bounds to the total absorption, just as the results derived with no excess may be considered as lower bounds to the true absorption.

\section{Absorption and phase delay along short atmospheric paths}

\subsection{Differential absorption and delay}

We first consider the atmospheric absorption and delay for short atmospheric paths. Locally the atmosphere can 
be considered to be both isothermal and isobaric, allowing us to directly use Eqs. (2) and (5), above. In Fig. 1 we plot the calculated atmospheric opacity and delay, divided by the precipitable water vapor content, for a short path. We assume a temperature of $270 \mathrm{~K}$ and a pressure of $750 \mathrm{mb}$, as might be expected for water vapor roughly $3 \mathrm{~km}$ above sea level. Thus these are conditions which are reasonably representative of water vapor above moderate altitude sites such as those currently used for millimeter interferometry.

The absorption spectrum displays the well known absorption features at 22 and $183 \mathrm{GHz}$. Otherwise the water vapor absorption throughout the centimeter and millimeter wavelength bands is dominated by the wings of a multitude of strong, higher-frequency lines. The magnitude of that absorption is very much dependent on the assumed shape of the line wings and any empirical "excess" absorption.

The delay spectrum at low frequencies is nearly flat, indicative of the well known fact that atmospheric water vapor is nearly dispersionless in the radio frequency region. There is a gradual increase in the delay as one approaches the millimeter region and small inflections in the vicinity of each water line. The variations in delay become quite pronounced above $500 \mathrm{GHz}$.

\subsection{Temperature dependence}

We have investigated the dependence of the opacity and the delay on temperature, and plot in Fig. 2 the corresponding partial derivatives of both quantities. Each is displayed as a fractional derivative, that is the fractional change in opacity (or delay) per Kelvin. As with Fig. 1, we illustrate the kinetic line shape and the effects of including the two forms of the "excess" opacity. These plots are based on a mean temperature of $270 \mathrm{~K}$ and a mean pressure of $750 \mathrm{mb}$. The values shown are insensitive to the choice of mean pressure. Changes in mean temperature of order $30 \mathrm{~K}$ produce changes of order $0.001 \mathrm{~K}^{-1}$ in $\tau^{-1} \partial \tau / \partial T$ and $0.0005 \mathrm{~K}^{-1}$ in $L^{-1} \partial L / \partial T$.

The partial derivative of the opacity shows considerable structure, with rapid variations in the vicinity of each water line. Generally the temperature coefficient is negative, with a value of about $-0.009 \mathrm{~K}^{-1}$ appropriate throughout much of the millimeter wavelength band. It is less negative in the cores of the 22 and $183 \mathrm{GHz}$ lines and is even somewhat positive at the very center of the 22 $\mathrm{GHz}$ line.

The partial derivative of the delay shows little structure at frequencies below $500 \mathrm{GHz}$. Throughout the millimeter band it is negative, with a value of about $-0.0036 \mathrm{~K}^{-1}$.

Radiometric monitoring schemes which use opacity to infer delay require similarity between the temperature coefficients of these quantities in order to be insensitive to temperature variations in the atmospheric water vapor.
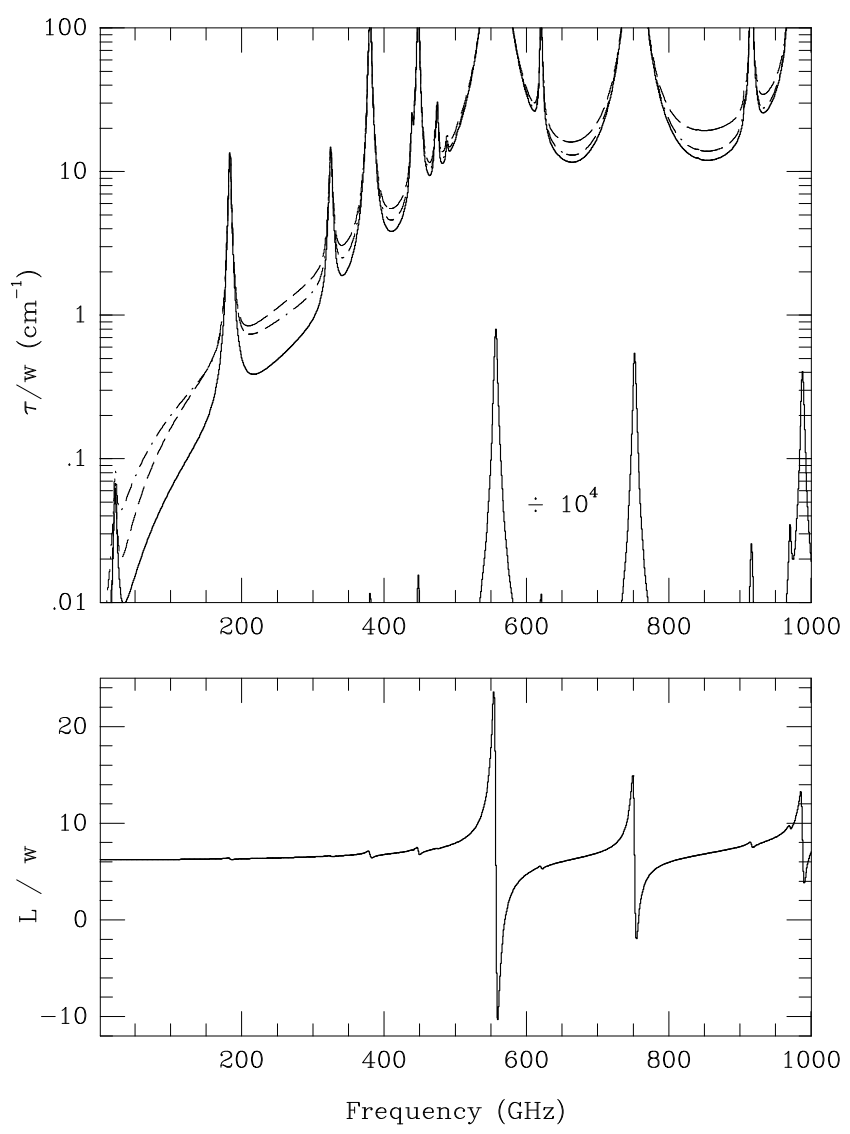

Fig. 1. Water vapor opacity and delay, each divided by the precipitable water vapor content, for a short atmospheric path at $T=270 \mathrm{~K}$ and $P=750 \mathrm{mb}$. The bold solid lines illustrate the kinetic line shape without any "excess" absorption. The thin dashed and dot-dashed lines show the effects of including respectively the Gaut and Reifenstein and Zammit and Ade forms of the "excess" opacity. The corresponding differences in $L / w$ are less than 0.015

However, a radiometer measures emission, not opacity, a fact which introduces an additional dependence on atmospheric temperature. The sky brightness due to atmospheric emission is given by

$$
T_{\mathrm{b}}=T\left(1-\mathrm{e}^{-\tau(T)}\right),
$$

where $T$ is the temperature of the atmospheric water vapor. The fractional partial derivative of $T_{\mathrm{b}}$ then contains two terms,

$$
\frac{1}{T_{\mathrm{b}}} \frac{\partial T_{\mathrm{b}}}{\partial T}=\frac{\tau \mathrm{e}^{-\tau}}{1-\mathrm{e}^{-\tau}} \frac{1}{\tau} \frac{\partial \tau}{\partial T}+\frac{1}{T} .
$$

In the optically thin limit this produces the term already discussed and shown in the upper part of Fig. 2, and an additional positive term proportional to $1 / T$. The effects of this additional term are shown in Fig. 3, which can then be compared with the lower part of Fig. 2. Thus there is good agreement of temperature coefficients throughout 
the millimeter wave atmospheric windows, and radiometric monitoring will provide a measure of atmospheric delay which is relatively insensitive to atmospheric temperature.
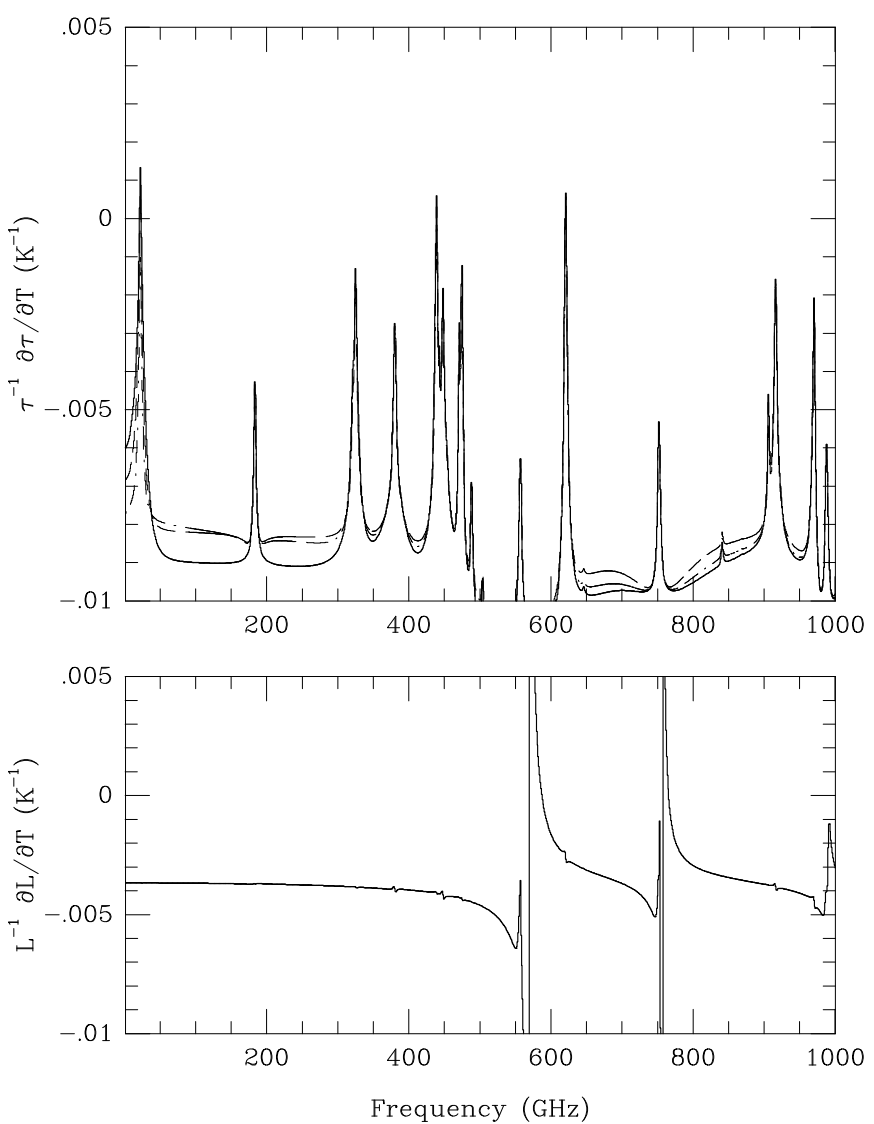

Fig. 2. Fractional changes in opacity and delay with temperature. The bold solid lines illustrate the kinetic line shape without any "excess" absorption. The thin dashed and dot-dashed lines include the Gaut and Reifenstein and Zammit and Ade forms of the "excess" opacity, respectively. These produce significant changes only in $\tau^{-1} \partial \tau / \partial T$

If the atmosphere is partially opaque, the variation due to changes in the opacity becomes less important and the temperature coefficient becomes less variable and more positive. This effect is illustrated in Fig. 4 for the case of

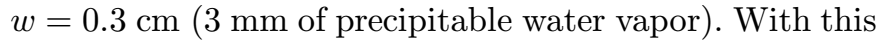
amount of water vapor the atmosphere is generally opaque at high frequencies but remains reasonably optically thin in the $3 \mathrm{~mm}$ wavelength band. Comparison with the lower part of Fig. 2 again illustrates the sensitivity to temperature fluctuations. In an atmosphere which is partially optically thick, radiometric monitoring is a less effective measure of atmospheric delay, in part due to an increased sensitivity to atmospheric temperature.

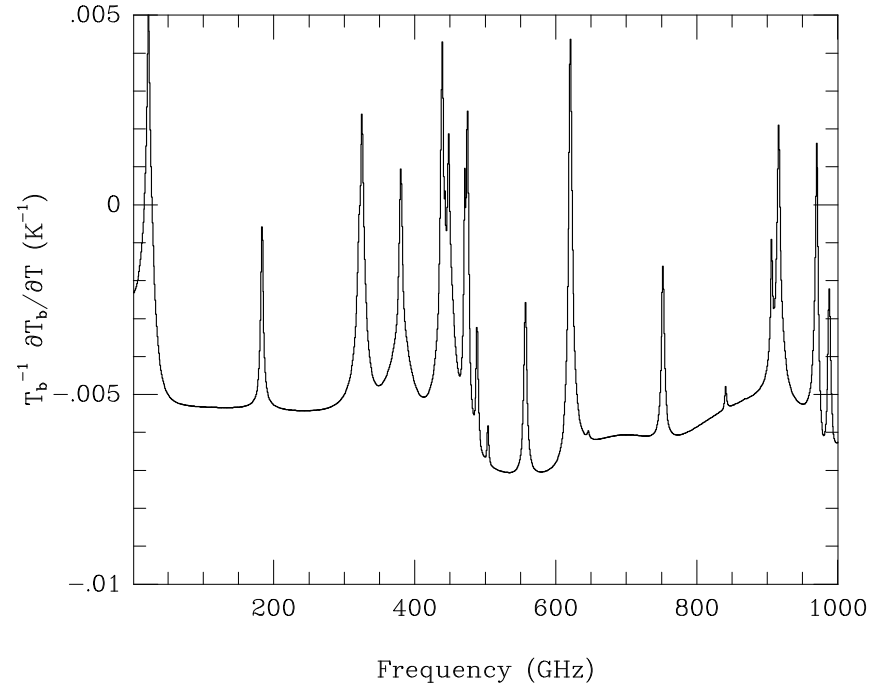

Fig. 3. Fractional change in sky brightness with temperature in the optically thin limit, based on the kinetic line shape

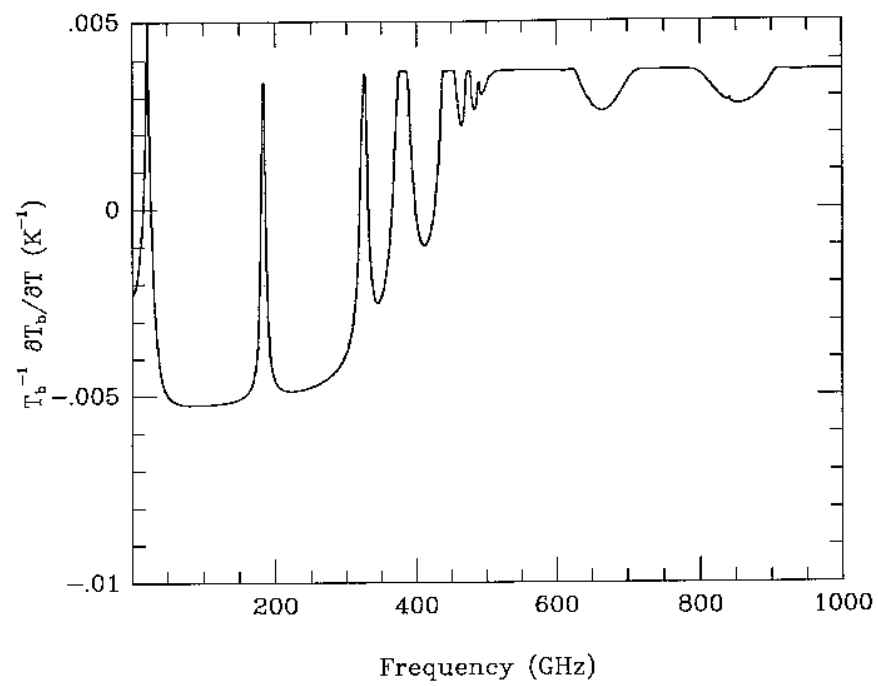

Fig. 4. Fractional change in sky brightness with temperature for $w=0.3 \mathrm{~cm}$. The kinetic line shape has been used

\subsection{Pressure dependence}

A similar analysis has been made of the dependence of opacity and delay on atmospheric pressure. Pressure is an important quantity since height of the atmospheric water vapor is not known a priori, and therefore the degree of pressure broadening is not known. This effect is shown directly in Fig. 5, where the partial derivative of the opacity is positive through much of band shown, with a typical value of $0.00133 \mathrm{mb}^{-1}$. This is directly illustrating the variation in line width with pressure. At higher pressures there is a larger line width and more opacity in the wings. This tendency is reversed only in the cores of the lines. 

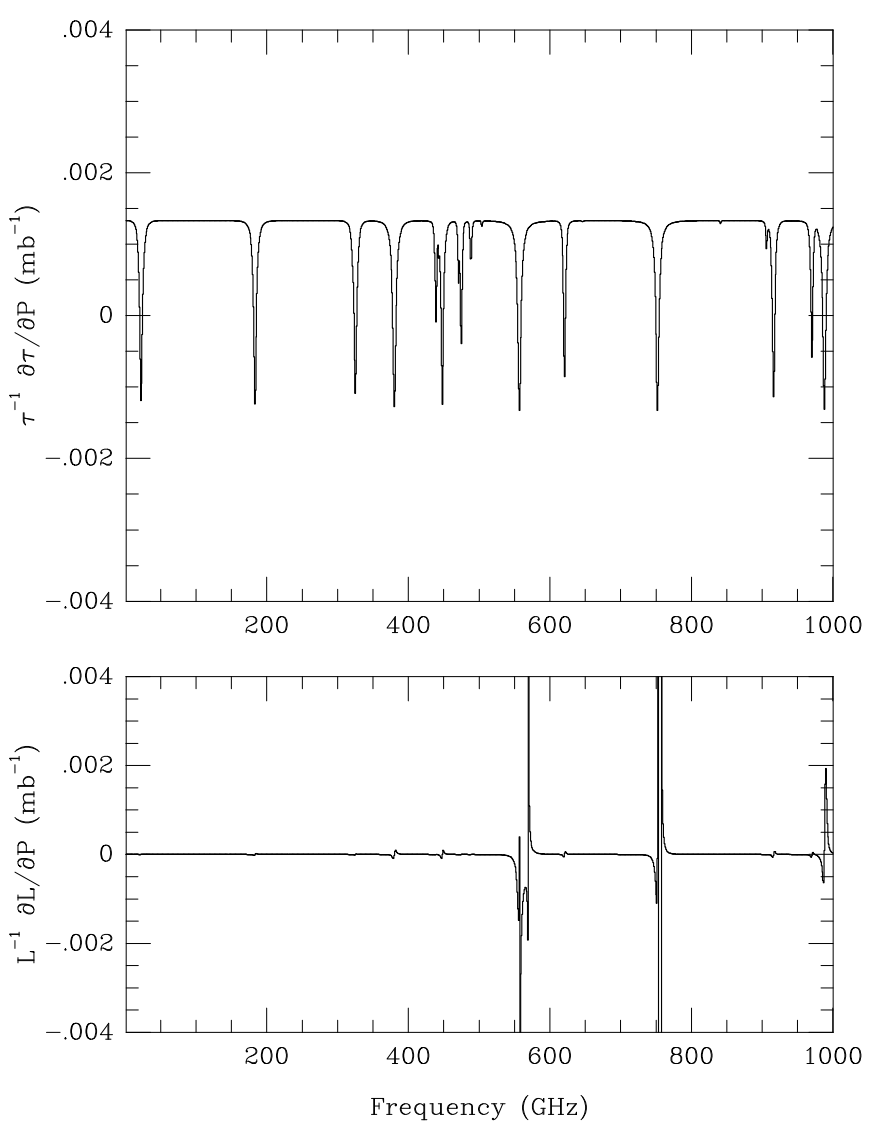

Fig. 5. Fractional changes in opacity and delay with pressure, based on the kinetic line shape

As line width increases with pressure, opacity is removed from the line cores as it is added in the line wings.

The delay shows little dependence on pressure except near the strongest high frequency lines. Thus radiometric monitoring is likely to be somewhat sensitive to pressure for most single frequency monitoring schemes. There are nulls in the partial derivative of the opacity with respect to pressure at the half power points of the absorption profiles. In principle these could be exploited by a narrow band monitor at the null, a broadband monitor spanning the null, or by a multiple frequency radiometer. Such a scheme could provide immunity to pressure variations.

\section{Layered atmospheric models}

We have also calculated opacity and delay for layered atmospheric models. In these models the pressure, temperature, and water vapor density profiles are specified and the opacity and delay are integrated vertically through the atmosphere. In Fig. 6 we show the results of a calculation incorporating a base pressure of $900 \mathrm{mb}$, a pressure scale height of $8 \mathrm{~km}$, a base temperature of $280 \mathrm{~K}$, a lapse rate of $6.5 \mathrm{~K} \mathrm{~km}^{-1}$, and a water vapor scale height of $2 \mathrm{~km}$. This puts the bulk of the water vapor at conditions near those used in Sect. 3.1, above, and as a result the spectra show good agreement with those in Fig. 1. The lines are slightly narrower in Fig. 6, indicating a mean effective pressure somewhat less than $750 \mathrm{mb}$.
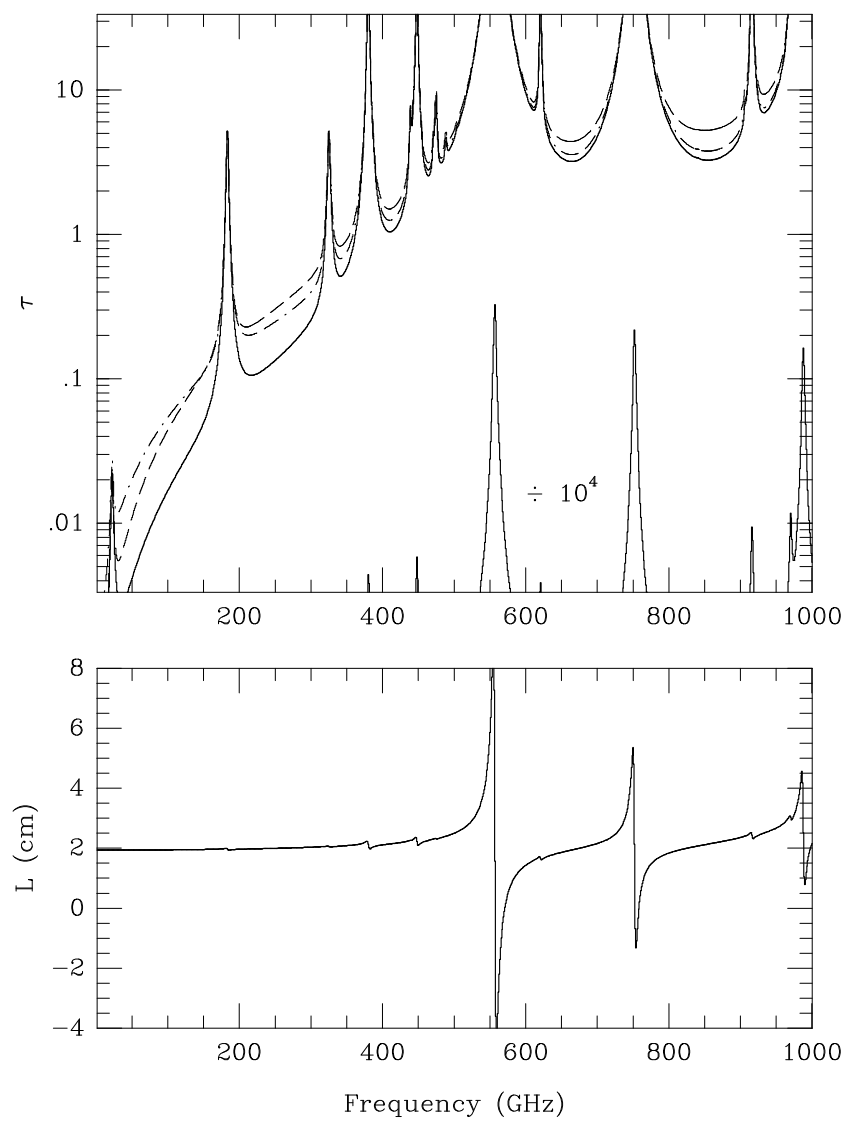

Fig. 6. Opacity and delay integrated vertically through the atmosphere, for a frequency of $225 \mathrm{GHz}$ and for $3.0 \mathrm{~mm}$ of precipitable water vapor. The bold solid lines are for the kinetic line shape, the thin dashed line includes the Gaut and Reifenstein excess, and the thin dot-dashed line includes the Zammit and Ade excess. Conditions are for a base pressure of $900 \mathrm{mb}$, base temperature of $280 \mathrm{~K}$, pressure scale height of $8 \mathrm{~km}$, water vapor scale height of $2 \mathrm{~km}$, and a lapse rate of $6.5 \mathrm{~K} \mathrm{~km}^{-1}$

The effectiveness of radiometric monitoring depends on the constancy of the ratio between the differential delay, $\mathrm{d} L(\nu)$, and the differential change in atmospheric sky brightness, $\mathrm{d} T_{\mathrm{b}}(\nu)$, both introduced by a small change in water vapor content, $\mathrm{dw}$. These quantities in turn depend on the detailed spatial distribution of the change in water vapor content and the temperature structure of the atmosphere. For a layered atmosphere, the sky brightness is given by

$$
T_{\mathrm{b}}=\int_{0}^{\infty} \alpha T \mathrm{e}^{-\tau^{\prime}} \mathrm{d} z=\int_{0}^{\tau} T \mathrm{e}^{-\tau^{\prime}} \mathrm{d} \tau
$$


The integral is evaluated from the ground up through the atmosphere and $\tau^{\prime}$ is the total opacity of the layers below the height $z$.

For the purpose of illustration we have evaluated the ratio $\mathrm{d} L(\nu) / \mathrm{d} T_{\mathrm{b}}(\nu)$ at a frequency of $225 \mathrm{GHz}$ for various scenarios. We show in Fig. 7 its variation with base temperature and pressure for a layered, optically thin atmosphere (in which case $\mathrm{d} L(\nu) / \mathrm{d} T_{\mathrm{b}}(\nu)=L(\nu) / T_{\mathrm{b}}(\nu)$ ). The base temperature and pressure are readily available meteorological parameters. We also illustrate its rather weak dependence on lapse rate, a quantity which is not as readily available. As an alternative, we consider an optically thin atmosphere in which the water vapor is assumed to be confined to a thin layer, and consider the dependence on the height of this layer above the ground. Such height information is also not readily available. While these scenarios are not exhaustive, they should be reasonable representative of atmospheric distributions which may be encountered. Where the atmosphere becomes optically thick, the value of $T_{\mathrm{b}}$ saturates, leading to larger (and differing) values for $L(\nu) / T_{\mathrm{b}}(\nu)$ and $\mathrm{d} L(\nu) / \mathrm{d} T_{\mathrm{b}}(\nu)$.
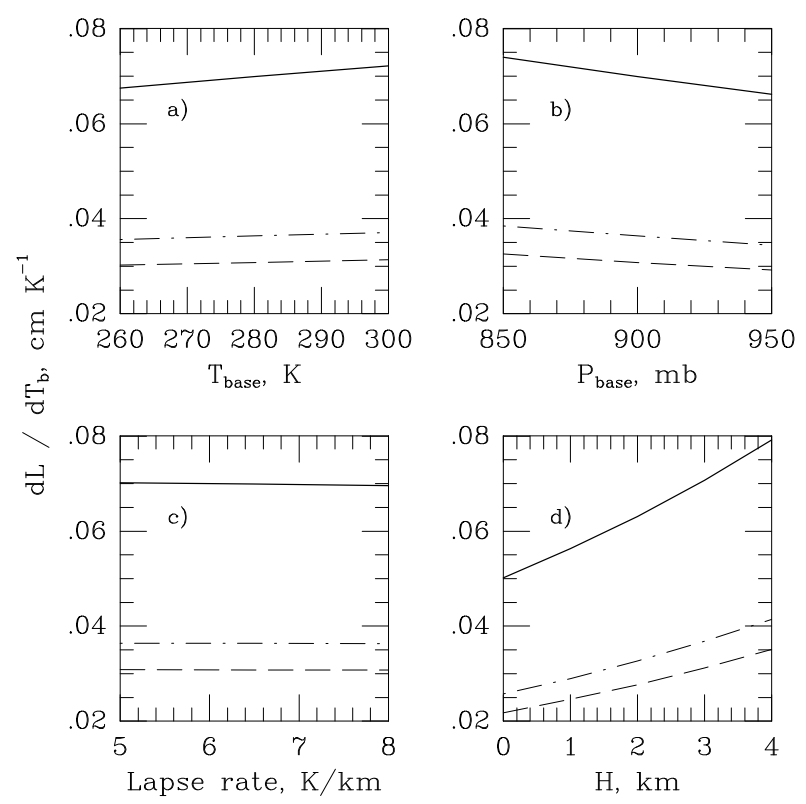

Fig. 7. Variation in $\mathrm{d} L(\nu) / \mathrm{d} T_{\mathrm{b}}(\nu)$ evaluated at $225 \mathrm{GHz}$ for optically thin atmospheres. Panels a), b) and c) illustrate a layered exponential atmosphere and the effects of varying the base pressure, base temperature, and lapse rate. Panel d) illustrates the situation in which the water vapor is confined to a single thin layer of variable height. Shown are the cases of no excess absorption (bold solid lines), the Gaut and Reifenstein excess (thin dashed lines), and the Zammit and Ade excess (thin dot-dashed lines)

\section{Radiometric monitoring}

\subsection{Single frequency radiometers}

Radiometers designed to operate at single, fixed frequencies may be used to monitor atmospheric phase delay. The choice of monitoring frequency does not depend on the frequency for which one wishes to know the delay, since the delays at various frequencies are interrelated in a well determined fashion (cf. Figs. 1 and 6). Instead the choice is determined by the magnitudes of systematic errors and by issues of sensitivity. The systematic errors for $225 \mathrm{GHz}$ were illustrated in Fig. 7. This behavior is representative of that expected for frequencies throughout the 200$300 \mathrm{GHz}$ atmospheric window. It is also reasonably representative of that expected in the $70-110 \mathrm{GHz}$ region $(\lambda \approx$ $3 \mathrm{~mm})$, except that the lower atmospheric opacity in the $3 \mathrm{~mm}$ band leads to smaller values of $T_{\mathrm{b}}$ and hence larger values of the ratio $\mathrm{d} L / \mathrm{d} T_{\mathrm{b}}$. Radiometric monitoring near the cores of any of the prominent water lines $(22,183$, $325 \mathrm{GHz}$ ) will lead to rather different systematic errors due to the different dependencies of the line opacity on atmospheric temperature and pressure. Near any of these lines there will be less of a problem associated with the form of the "excess" absorption, since any such excess will represent a smaller fraction of the total opacity.

The variation in the ratio $\mathrm{d} L(\nu) / \mathrm{d} T_{\mathrm{b}}(\nu)$ for a thin layer of water vapor of variable height above the ground is shown in Fig. 8 for a variety of frequencies, both on and off water lines. The results shown assume a base pressure of $900 \mathrm{mb}$, a base temperature of $280 \mathrm{~K}$, and a lapse rate of $6.5 \mathrm{~K} \mathrm{~km}^{-1}$. In all cases the atmosphere is taken to be optically thin. The plots for 90,225 , and $345 \mathrm{GHz}$ show positive slopes which are largely due to the positive pressure coefficient of $\tau$ (cf. Fig. 5). The plots for 22 and $183 \mathrm{GHz}$ show negative slopes due to the sign reversals in $\partial \tau / \partial P$ in the line cores. At $186 \mathrm{GHz}$ the pressure coefficient is near zero, leading to a weak dependence on the height of the water layer. These results are illustrative of situations which may be encountered, but actual atmospheric variations may be more complex. In particular, the temperature variation with height may not be describable by a simple lapse rate. As discussed in Sect. 3.2, the relationship between delay and atmospheric brightness is significantly more sensitive to temperature in the line cores (e.g. 22, 183, $186 \mathrm{GHz}$ ) than elsewhere. This effect is not obvious in Fig. 8 since, for the simple temperature structure and lapse rate chosen, the effects of the temperature variation are small and somewhat cancel those associated with the pressure variation.

Figure 8 shows that in regions of good atmospheric transparency $(22,90,225$, and $345 \mathrm{GHz})$, the uncertainty regarding the existence and magnitude of the "excess absorption" will dominate the a priori absolute calibration of a single frequency radiometric monitoring scheme. Zivanovic (1992) calculated a ratio $\mathrm{d} \phi / \mathrm{d} T_{\mathrm{b}} \approx 280^{\circ} \mathrm{K}^{-1}$ 

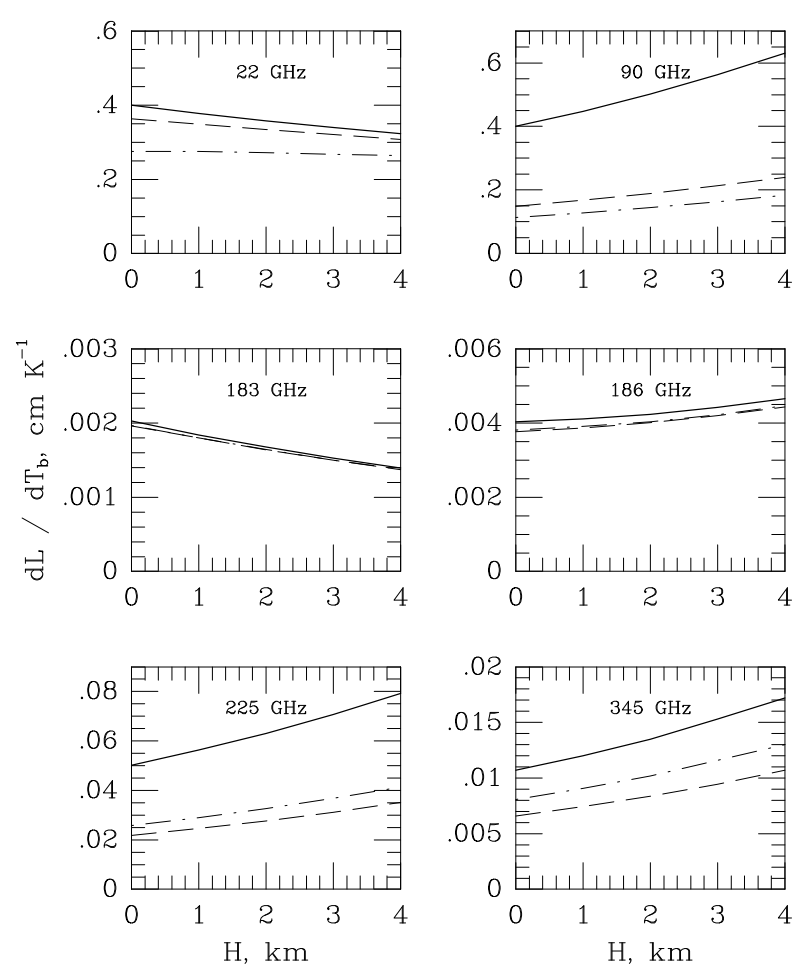

Fig. 8. The ratio of changes in delay to changes in sky brightness for various frequencies in the optically thin limit, assuming a thin layer of water vapor of variable height. For reasonable values of the water vapor content, $w$, the more opaque regions $(183,186,345 \mathrm{GHz})$ will be somewhat optically thick, leading to larger values of $\mathrm{d} L / \mathrm{d} T_{\mathrm{b}}$. The bold solid lines refer to no excess absorption, the thin dashed lines to the Gaut and Reifenstein excess, and the thin dot-dashed lines to the Zammit and Ade excess

at $90 \mathrm{GHz}$, corresponding to $\mathrm{d} L / \mathrm{d} T_{\mathrm{b}}=0.26 \mathrm{~cm} \mathrm{~K}^{-1}$, which is very close to the observed ratio at Hat Creek using the Berkeley-Illinois-Maryland Association (BIMA) interferometer (Plambeck 1996). In Fig. 8 this value falls approximately midway between the curve representing no "excess absorption" and those representing the two empirical forms of the excess. This supports our assertion in Sect. 2.4 that these represent limiting cases, with the true absorption falling somewhere in between. Thus if the kinetic line shape is adopted, the excess absorption appears to be only about half of that suggested by Gaut and Reifenstein or Zammit and Ade. It remains to be seen whether this property is constant under varying atmospheric conditions.

The ability to detect atmospheric radiometric fluctuations at various frequencies is intimately connected with the characteristics of the receiver systems employed. With high quality cryogenic mixers the receiver sensitivity is not a major concern, and it is reasonable to expect to be able to monitor delay fluctuations of order $0.005 \mathrm{~cm}$ or better in a 1 second integration time with a bandwidth of $1 \mathrm{GHz}$.
Gain stability is often a more significant issue, particularly at frequencies where the atmosphere is highly transparent and the brightness fluctuations are small. Zivanovic (1992) has estimated that gain stabilities of order one part in $10^{4}$ are needed for effective radiometric monitoring at 90 $\mathrm{GHz}$ at Hat Creek. At $225 \mathrm{GHz}$ the atmosphere is more opaque. The brightness fluctuations are correspondingly larger and gain stabilities of order one part in $10^{3}$ are sufficient. Gain stability is not generally a major problem in the line cores, except for the rather weak $22 \mathrm{GHz}$ line. Variations in ground pickup are also a concern, again particularly at frequencies where the atmospheric brightness fluctuations are small.

The advantages of detecting brightness fluctuations at frequencies where they are relatively large are partially offset by the effects of saturation. Significant atmospheric opacity implies a non-linear relationship between $L$ and $T_{\mathrm{b}}$, which may be difficult to interpret, and a reduction in the brightness fluctuations for $\tau \gtrsim 1$.

\subsection{Multiple frequency radiometers}

Radiometric monitoring may be conducted at several frequencies simultaneously, and there are a large number of possible choices for which frequencies to use. Individually the radiometric signal from each frequency will be subject to the systematic uncertainties discussed above. However, such signals may be used jointly to discriminate against systematic effects. For example, monitoring at 186 and $225 \mathrm{GHz}$ could yield independent determinations of water vapor content (and thereby, delay) and the pressure at the absorbing layer. Another possible use is discrimination between water vapor and condensed water (Crane 1976). The extinction by droplets of liquid water varies rapidly at low frequencies, and this may be used to discriminate between the presence of water vapor and condensed water. However, unless the number of frequencies monitored is quite large, it will difficult to determine all possible parameters of interest (pressure, temperature, vapor content, and liquid content).

\section{Summary}

Radiometric monitoring of atmospheric water vapor can be an effective method of measuring the phase fluctuations relevant to millimeter-wavelength interferometry. However, careful attention must be given to systematic effects which may enter into the determination of the proper ratio between atmospheric brightness and phase delay. It appears that, at many frequencies, pressure variations associated with different height distributions of water vapor may be the principal source of uncertainty. This effect is small near the half-power points in the line profiles, but is of significant magnitude both in the line cores and in the line wings. Also of importance are variations in the temperature of the water vapor. The dependence of the 
absorption spectrum on temperature is complex, but broadly speaking the ratio between atmospheric brightness and phase delay is least dependent on temperature in the inter-line regions. Uncertainties in line shape profiles or in the form of any "excess" (continuum) absorption affect our a priori understanding of the brightness to phase delay ratio, particularly at frequencies where the atmospheric opacity is low. But unless the line shapes vary with time or the continuum has some unknown dependence on temperature or pressure, such uncertainties may not limit our ability to monitor atmospheric variations once a proper ratio has been determined.

Acknowledgements. This work has been supported in part by the National Science Foundation under grants AST 91-17740 and AST 93-20239 and by NASA under grant NAGW-4724.

\section{References}

Benedict W.S., Kaplan L.D., 1959, J. Chem. Phys. 30, 388

Ben-Reuven A., 1969, Adv. Atom. Mol. Phys. 5, 201

Burch D.E., 1968, J. Opt. Soc. Amer. 58, 1383

Crane R.K., 1976, in: Meth. Exper. Phys. 12B, Meeks M.L. (ed.), pp. 177-185

Danese L., Partridge R.B., 1989, ApJ 342, 604

Falcone V.J.Jr., 1969, Appl. Opt. 8, 2362

Gaut N.E., 1968, MIT RLE Tech. Rep. 467

Gaut N.E., Reifenstein E.C. III, 1971, Environmental Res. Tech. Rep. No. 13, Lexington, Massachusetts

Gross E.P., 1955, Phys. Rev. 97, 395

Herzberg G., 1945, Infrared and Raman Spectra of Polyatomic Molecules, pp. 506-508

Liebe H.J., 1981, Radio Sci. 16, 1183

Liebe H.J., 1984, Int. J. Infrared Mill. Waves 5, 207

Liebe H.J., 1985, Radio Sci. 20, 1069

Plambeck R.L., 1996, (personal communication)

Poynter R.L., Pickett H.M., 1984, Submillimeter, Millimeter, and Microwave Spectral Line Catalog, JPL Publication 8023, Revision 2

Van Vleck J.H., 1947, Phys. Rev. 71, 425

Van Vleck J.H., Weisskopf V.F., 1945, Rev. Mod. Phys. 17, 227

Waters J.W., 1976, in: Meth. Exper. Phys. 12B, Meeks M.L. (ed.), pp. 142-176

Zammit C.C., Ade P.A.R., 1981, Nat 293, 550

Zivanovic S.S., 1992, Ph. D. thesis, University of California, Berkeley 